ISSN: 2394-2258

Available at http://scientificadvances.co.in

DOI: http://dx.doi.org/10.18642/ijamml_7100122024

\title{
FUZZY CLUSTERING IMAGE INPAINTING ALGORITHM BASED ON MODIFIED CUCKOO SEARCH
}

\section{Duanliang Cao}

School of Information and Science Technology, Jinan University, Guangzhou, 510632, P. R. China

\begin{abstract}
Fuzzy C-means (FCM) algorithm is a widely used clustering, however, it is influenced by the initial cluster centers, and is easy to fall into local opitma. In order to overcome this drawback, we propose a modified cuckcoo search (MCS) which changes the detection probability $P$ from a constant value to a varible number of iterations decreases. This will not only improve the quality of the population, but also ensure the covergence of the algorithm. Therefore, we can use the MCS algorithm to generate the FCM clustering centers and avoid the local optimal problem of FCM. The proposed algorithm has better clustering effect and faster running speed. In this paper, MCS_FCM algorithm classifies the image pixels into a number of categories according to the similarity principle, making the similar pixels clustering into the same category as possible. According to the provided gray value of the pixels to be inpainted, we calculate the category whose distance is the nearest to the inpainting area and this category is to be inpainting area, and then the inpainting area is restored by the modified CDD model to realize image inpainting.
\end{abstract}

\footnotetext{
${ }^{*}$ Corresponding author.

E-mail address: duanliangcao@163.com (Duanliang Cao).
}

Copyright () 2019 Scientific Advances Publishers

2010 Mathematics Subject Classification: 00A06, 68 U10.

Submitted by Jianqiang Gao.

Received November 16, 2018 
Keywords: image inpainting (restoration), modified cuckoo search (MCS), fuzzy C-means (FCM), MCS_FCM, modified CDD model.

\section{Introduction}

Image inpainting is a technology for restoring the damaged parts of an image by referring to the information from the undamaged parts to make the restored image look complete continuous and natural. Bertalmio et al. [1] firstly proposed a model based on isophote to continuously propagate the undamaged information into the destroyed region. Chan and Shen [3] proposed a variational model based on total variation (TV) to repaired the destroyed information. Because TV model produced ladder effect, some scholars proposed CDD model [4]. Wang et al. proposed an modified cuckoo search optimization algorithm [5]. In this paper, we proposed the algorithm to identify the damaged area and also combined the modified CDD model to realize image inpainting.

The MCS_FCM algorithm to automatically identified the damaged area and also combined the modified CDD model to realized image inpainting. In Section 2, we presented an image inpainting algorithm based on clustering analysis of MCS_FCM. And in Section 3, the experiment of the proposed method is implemented. We end this paper with some conclusions in Section 4.

\section{Theoretical Basis of MCS_FCM Identify Damaged Areas}

\subsection{Modified cuckoo search algorithm}

Cuckoo search (CS) was developed by Yang and Deb [6]. This paper proposed an MCS, which mainly modified the discovery probability $P$. The formula for $P$ as a function of the number of iterations is:

$$
P=P_{\min }+\left(P_{\max }-P_{\min }\right) \times((G-g) / G),
$$


where $G$ represents the maximum number of iterations, $P_{\min }$ and $P_{\max }$ represent the minimum and maximum values of $P$. The MCS algorithm it can also effectively solve the problem that FCM [7] is easy to fall into local optimal value. The following fitness function:

$$
f=n / J(U, A)=1 / \sum_{j=1}^{n} \sum_{i=1}^{c} u_{i j}^{m} d_{i j}^{2},
$$

where $J(U, A)$ is the sum of the objective function values of each pixel. The steps of algorithm as follows:

(1) Parameter initialization. Including $n, d, P_{\min }, P_{\max }, G, c, m$.

(2) Randomly initialized cluster centers, the initial bird nest position.

(3) Calculated the fitness function values.

(4) Calculated the fitness function value of the new bird's nest again.

(5) Compare the new function value with the previous generation, and change the bird's nest position, otherwise it will not change.

(6) Compared the random number $r(r \in[0,1])$ with $P$. Finally, the optimal bird's nest position is retained.

(7) If $i>$ MAXLEN, outputted nest position, otherwise return to Step 3.

(8) Obtained the final bird nest position, and outputted the result.

\subsection{Modified CDD model}

\subsubsection{Model establishment}

The modified CDD model is:

$$
\frac{\partial u}{\partial t}=\nabla[g(|\kappa|) \nabla u]+\lambda_{D}\left(u_{0}-u_{1}\right) .
$$


Introducing a weight function to the model (5)

$$
\left\{\begin{array}{l}
\frac{\partial u}{\partial t}=f(|\nabla u|) \cdot \nabla \cdot[g(|\kappa|) \nabla u]+\lambda_{D}\left(u_{0}-u\right), \\
g(|\kappa|) \text { is non-negative increasing function } \\
f(|\nabla u|) \text { is non-negative increasing function } \\
\kappa=\nabla \cdot\left[\frac{\nabla u}{|\nabla u|}\right] .
\end{array}\right.
$$

\subsubsection{Discretization of the model}

As shown in Figure 1, $O$ is the inpainting pixel point, and $\Lambda_{O}=\{N, S, W, E\}$ means four neighbourhood points set of $O$.

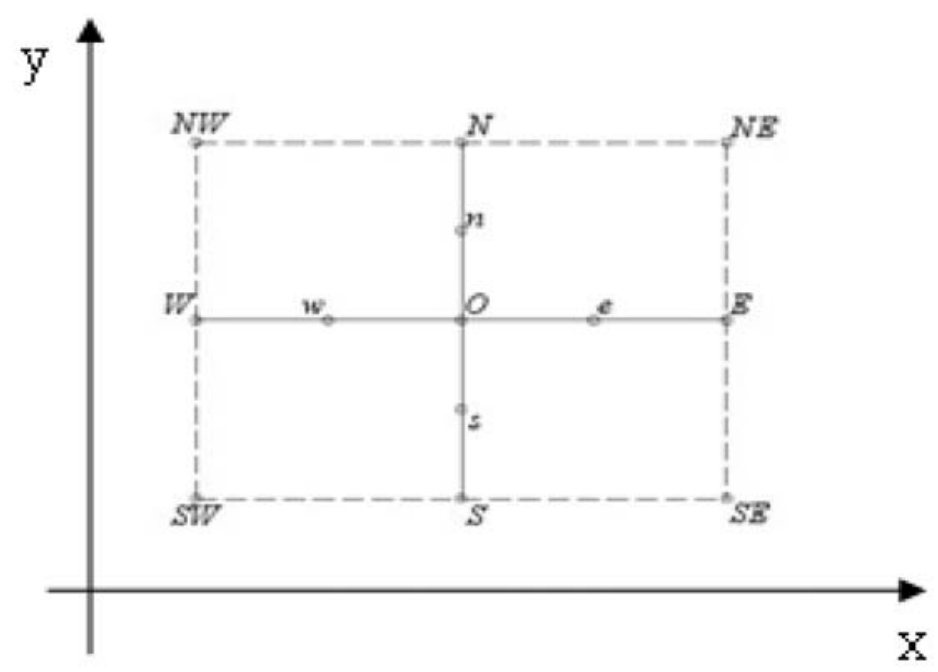

Figure 1. Inpainting pixel points and neighbour fields.

Let $\quad v=\left(\nu^{1}, \nu^{2}\right)=g(|\kappa|) \nabla u$ and take performance about central difference numerical method for divergence,

$$
\nabla \cdot \nu=\frac{\partial \nu^{1}}{\partial x}+\frac{\partial \nu^{2}}{\partial y} \approx \frac{v_{e}^{1}-\nu_{w}^{1}}{h}+\frac{\nu_{n}^{2}-\nu_{s}^{2}}{h}
$$


Then,

$$
\omega_{q}=f\left(\left|\nabla u_{q}\right|_{\varepsilon}\right) g\left(\left|\kappa_{q}\right|\right), h_{Q}=\frac{\omega_{q}}{\lambda_{D}(O)+\sum_{Q \in \Lambda_{O}, q \in \Lambda} \omega_{q}}, h_{O}=\frac{\lambda_{D}(O)}{\lambda_{D}(O)+\sum_{Q \in \Lambda_{O}, q \in \Lambda} \omega_{q}} .
$$

Use Gauss-Jacobi iteration method to search the optimum:

$$
u^{n}(i, j)=\sum_{Q \in \Lambda, q \in \Lambda} h_{Q}^{(n-1)} u_{Q}^{(n-1)}+h_{O}^{(n-1)} u_{O}(i, j)
$$

The modified CDD model inpainting algorithm:

Initialized $u^{(0)}(i, j)$, generally we set $u^{0}(i, j)=u(i, j)$ :

For $n=1,2, \ldots$

$$
\begin{aligned}
& \text { For } i=1,2, \ldots, M \\
& \text { For } j=1,2, \ldots, N
\end{aligned}
$$

By the use of formula (8), calculate $\omega_{q}, h_{Q}, h_{O}$, respectively, the $(i, j)$ as the center of them, then use formula (9) to calculate.

End

End

\section{End}

\section{Computer Experiment}

The experiment is based on the MATLAB platform. All experiments are run on Core i7 CPU, and Windows 7 operating system. In the early stage of the algorithm iteration, $P$ should take a larger value. As the progress of algorithm, $P$ should be gradually reduced. Finally, we used the improved signal-to-noise (ISNR) as the objective metric standard of the quantity of the inpainting image, the definition of ISNR as: 


$$
I S N R=10 \cdot \log \left\{\frac{\sum_{x=1}^{M} \sum_{y=1}^{N}\left[\hat{I}(x, y)-I^{0}(x, y)\right]^{2}}{\sum_{x=1} \sum_{y=1}\left[\hat{I}(x, y)-I^{0}(x, y)\right]^{2}}\right\},
$$

where $\hat{I}(x, y)$ is the original gray image, $I^{0}(x, y)$ is the damaged image, and $I(x, y)$ is the recovered image.

\subsection{Scratch inpainting}

In the experiment, we set $C=9$, to initialized clustering centers

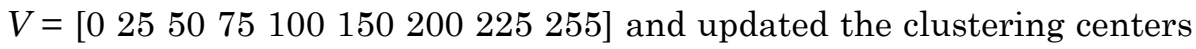
based on MCS_FCM, obtained the clustering centers $V^{\prime}=[28.6218$, 50.2181, 80.2287, 111.0351, 14.5698, 171.0493, 197.4505, 216.6581, 253.2535]. Finally, combined CDD model inpainting algorithm with MCS_FCM identifying the damaged areas, and get the results as follows. 
The damaged image

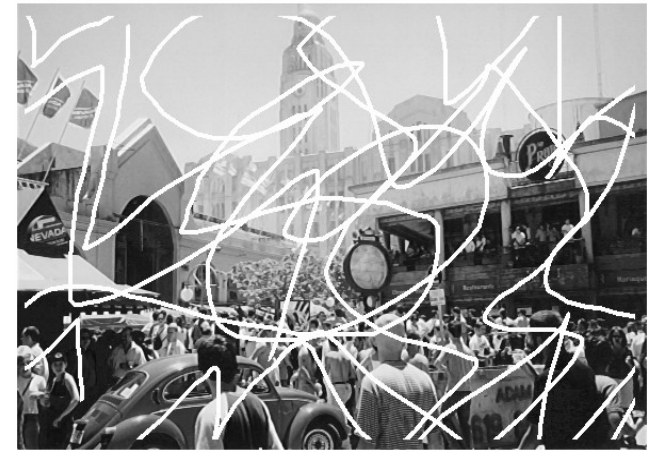

The damaged image

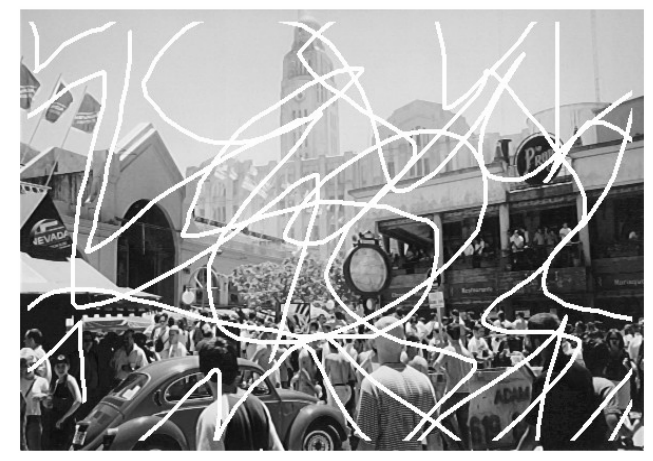

The deletion region by MCS_FCM

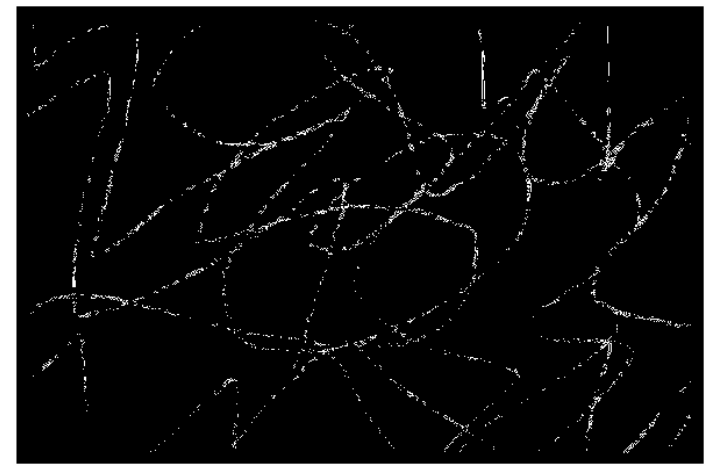

Repaired image with modified CDD model

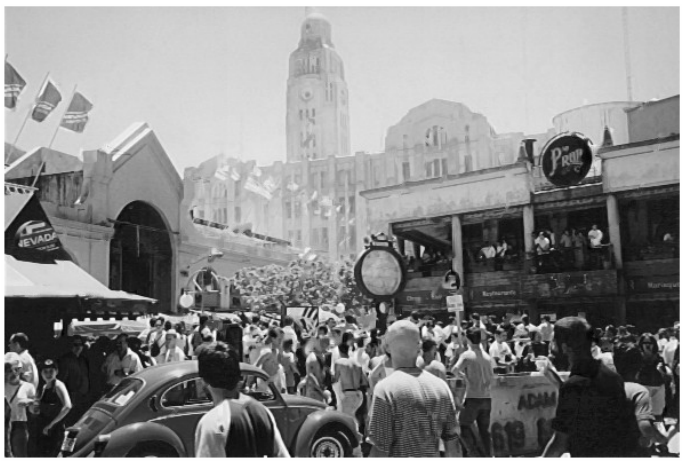

Figure 2. The result based on MCS_FCM, T = 0.91731, ISNR = 15.2988. 
The damaged image

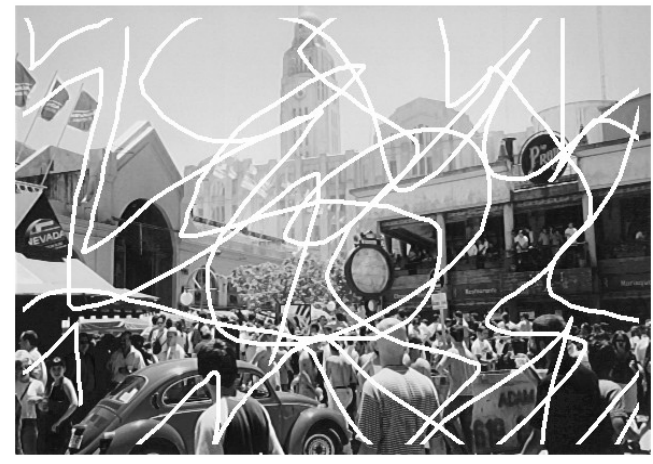

The damaged image

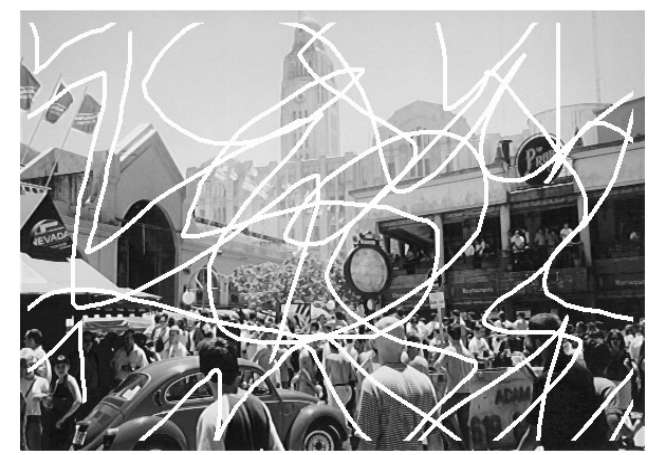

The deletion region by FCM

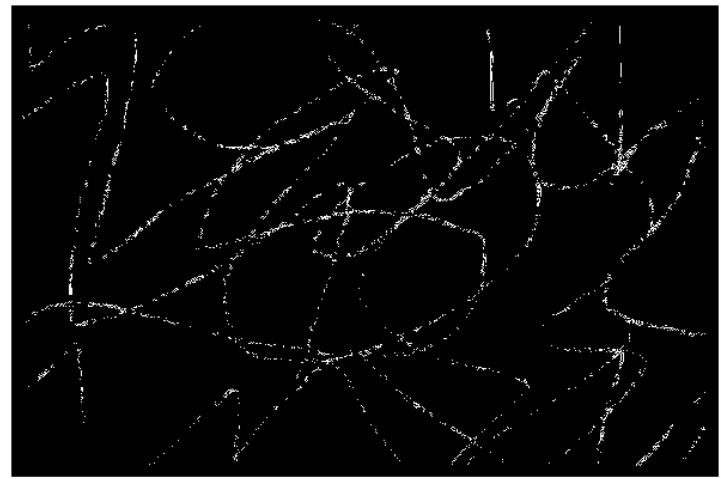

Repaired image with modified CDD model

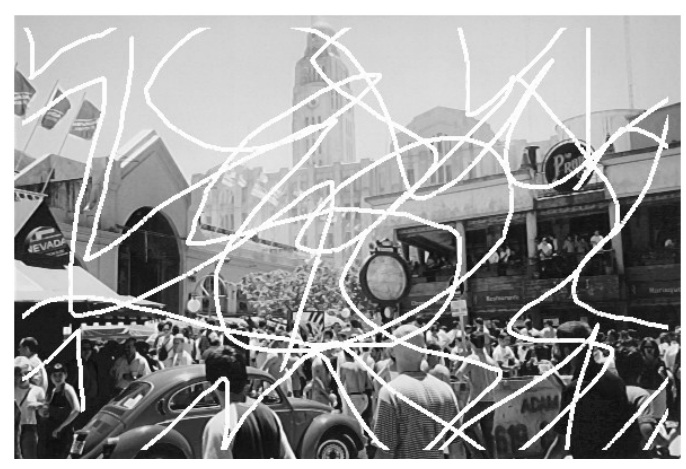

Figure 3. The result based on FCM, $\mathrm{T}=2.4639, \mathrm{ISNR}=0.34366$. 
Compared with the article [8], MCS_FCM has better repair effect and ISNR is bigger.

\subsection{The big block area inpainting}

In the experiment, we set $C=12, P_{\max }=0.5, P_{\min }=0.1$, to

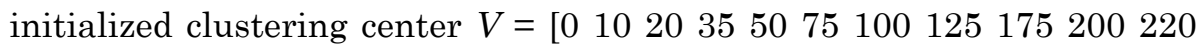
255] and updated the clustering centers based on MCS_FCM, obtained the clustering centers $V^{\prime}=[2.4434,32.8452,55.7445,83.6333,104.6469$, 126.9488, 150.063, 166.623, 180.1456, 194.4942, 214.7961, 244.9137]. Finally, combined CDD model inpainting algorithm with MCS_FCM identifying the damaged areas, and get the results as follows. 
The damaged image

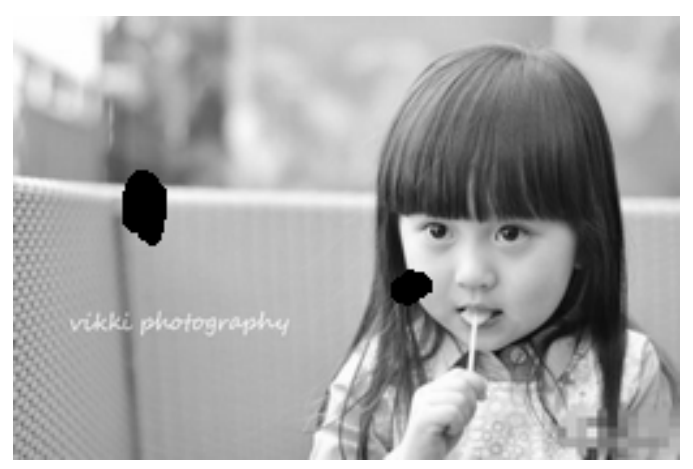

The damaged image

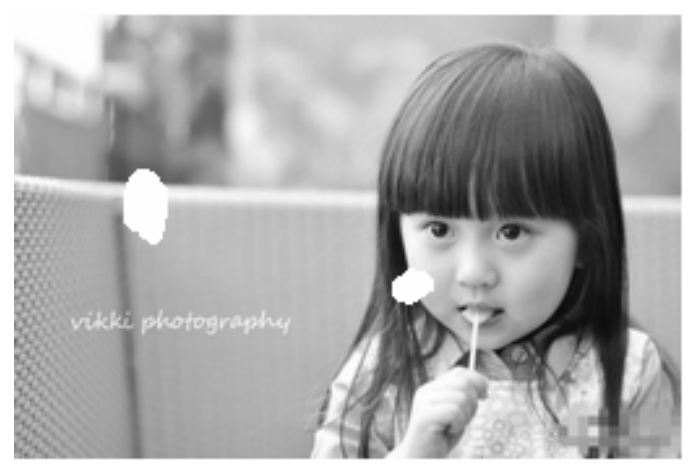

The deletion region by MCS_FCM

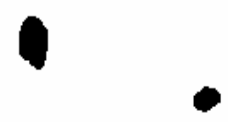

Repaired image with modified CDD model

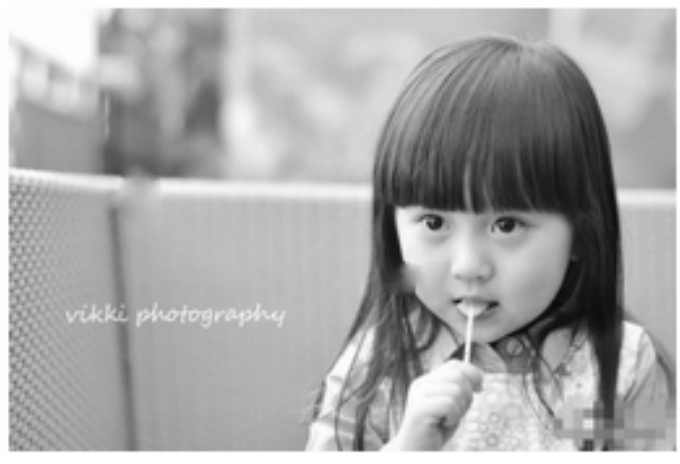

Figure 4. The result based on MCS_FCM, T $=0.15527$, ISNR $=40.3526$. 
The damaged image

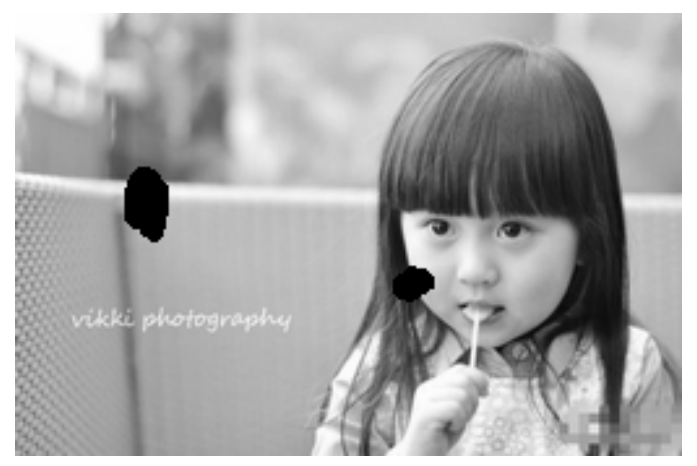

The damaged image

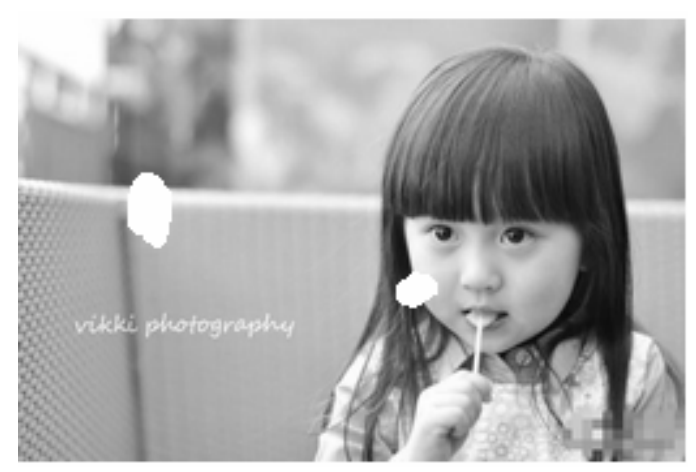

The deletion region by $\mathrm{FCM}$

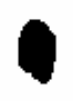

Repaired image with modified CDD model

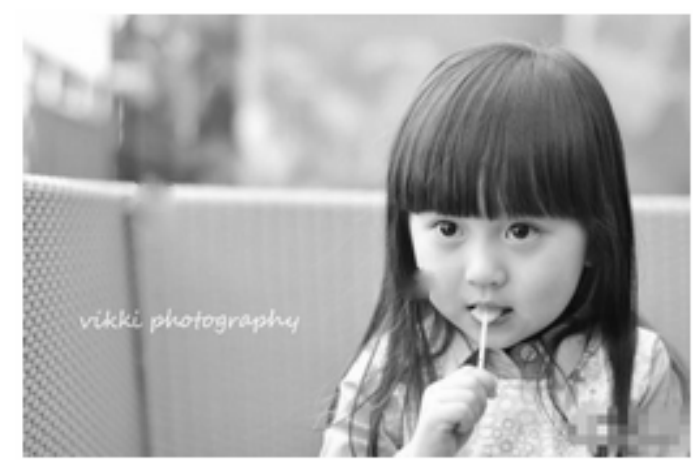

Figure 5. The result based on FCM, $\mathrm{T}=0.2568, \mathrm{ISNR}=37.0337$. 
Compared with the article [8], MCS_FCM has better repair effect and ISNR is bigger.

\section{Conclusion}

In this paper, we proposed fuzzy clustering image inpainting algorithm based on MCS_FCM. From the above experimental analysis, it can be seen that the damage area of the extracted image based on MCS_FCM is more accurate than the FCM clustering algorithm, and the final image breakage area will not fall into the local solution and the damaged area will be more accurate, so that with the modified CDD model to repair the image can get better results. Experimental results proved the superiority of the proposed method. As combined with the inpainting model, we can realize image inpainting function.

\section{References}

[1] M. Bertalmio, G. Sapiro, V. Caselles and C. Ballester, Image Inpainting, Proceeding of SIGGPAPH ACM, New York, ACM Press (2000), 417-424.

[2] L. I. Rudin, S. Osher and E. Fatemi, Nonlinear total variation based noise removal algorithms, Physica D: Nonlinear Phenomena 60(1-4) (1992), 259-268.

DOI: https://doi.org/10.1016/0167-2789(92)90242-F

[3] J. H. Shen and T. F. Chan, Mathematical models for local nontexture inpaintings, Society for Industrial and Applied Mathematics 62(3) (2001), 1019-1043.

DOI: https://doi.org/10.1137/S0036139900368844

[4] J. H. Shen, S. H. Kang and T. F. Chan, Euler's elastica and curvature-based inpainting, Society for Industrial and Applied Mathematics 63(2) (2003), 564-592.

DOI: https://doi.org/10.1137/S0036139901390088

[5] J. Wang, B. H. Zhou and S. D. Zhou, An improved cuckoo search optimization algorithm for the problem of chaotic system parameter estimation, Computational Intelligence and Neuroscience 2016, Article ID 2959370, pages 1-8.

DOI: http://dx.doi.org/10.1155/2016/2959370

[6] Xin-She Yang and Suash Deb, Cuckoo Search via Lévy flights, World Congress on Nature \& Biologically Inspired Computing (NaBic), 2009. 
[7] S. Miyamoto, H. Ichihashi and K. Honda, Algorithms for Fuzzy Clustering: Methods in C-means Clustering with Applications, Springer, New Yorks, NY, USA, 2008.

[8] Jiansheng Liu, Hui Liu, Shangping Qiao and Guangxue Yue, An automatic image inpainting algorithm based on FCM, The Scientific World Journal 2014, Article ID 201704, pages 1-10.

DOI: http://dx.doi.org/10.1155/2014/201704 